\title{
Effects of combining ultraviolet and mild heat treatments on enzymatic activities and total phenolic contents in pineapple juice
}

\begin{abstract}
Ultraviolet (UV) is able to inactivate most microorganisms in fruit juices with a low absorption coefficient but its effect is limited in inactivating undesired enzymes. The aim of this study was to overcome limitation of ultraviolet light (UV) by combining mild heat with UV. Pineapple juice was treated with mild heat (temperature: 50,55 and $60{ }^{\circ} \mathrm{C}$; holding time: 10, 20 and $30 \mathrm{~min}$ ) and subsequently exposed to $\mathrm{UV}(5.61,7.55$ and $11.23 \mathrm{~mJ} \cdot \mathrm{cm}-2)$. The effects of these combined treatments on pectin methylesterase (PME), bromelain activities and total phenolic content (TPC) were determined. Both enzymatic activities were reduced by mild heat but not by UV treatment. Increasing holding time and UV dosage led to higher depletion of TPC. Treating pineapple juice with mild heat at $55^{\circ} \mathrm{C}$ for $10 \mathrm{~min}$ and UV at 5.61 $\mathrm{mJ} \cdot \mathrm{cm}-2$ decreased PME by $60.53 \%$ whilst retaining $61.57 \pm 0.21 \%$ and $72.80 \pm 0.33 \%$ of bromelain and TPC, respectively. Industrial relevance: As opposed to traditional heat pasteurisation, ultraviolet (UV) treatment has the potential to produce pineapple juice with added value, such as high amount of health benefiting phenols and bromelain. Despite being known for being economically feasible, this technology is not widely adapted by the industry due to its inability to inactivate pectin methylesterase (PME). To overcome the limitation of $\mathrm{UV}$, mild heat $(\mathrm{MH})$ is introduced as hurdle technology. This study demonstrates that combining UV and MH could be able to effectively inactivate the PME in pineapple juice whilst preserving relatively high amount of bromelain and phenols.
\end{abstract}

Keyword: Ultraviolet; Mild heat; Pineapple juice; Pectin methylesterase; Bromelain; Total phenolic contents 